\title{
Probing structural dynamics of biomolecules in solution at high resolution via time-resolved small- and wide-angle $x$-ray scattering \\ P. Anfinrud ${ }^{1}$, H. S. Cho ${ }^{2}$, F. Schotte ${ }^{3}$, V. Stadnytskyi ${ }^{4}$ \\ ${ }^{1}$ National Institutes of Health ${ }^{2}$ National Institutes of Health, ${ }^{3}$ National Institutes of Health, ${ }^{4}$ National \\ Institutes of Health \\ anfinrud@nih.gov
}

To understand how biomolecules execute their designed function, it is crucial to know not only their native structures, but also how those structures evolve over time. The time-dependent x-ray scattering signature unique to a biomolecule is accompanied by scattering from its hydration shell, the surrounding buffer, the sample container, and anything else in the path between the x-ray source and the beamstop. When the sample undergoes a laser-triggered temperature jump, the scattering signature from both the biomolecule and its surrounding solvent can change. To isolate the biomolecule contribution, it is quite helpful to characterize the SAXS/WAXS pattern over a broad range of $\mathrm{q}$ and temperatures. To that end, we have helped develop on the BioCARS beamline at the APS the infrastructure needed to probe structural dynamics of biomolecules in solution at high resolution in both space and time by acquiring time-resolved small- and wide-angle x-ray scattering patterns (SAXS/WAXS) following either photo activation of a chromophore or a laser-induced temperature jump. This infrastructure includes home-built instrumentation that allows us to rapidly scan and precisely control the sample temperature over a range spanning $20-120{ }^{\circ} \mathrm{C}$, and probe time-resolved changes in the scattering signature following a temperature jump with time resolution down to the $<10 \mathrm{~ns}$ duration of the laser pulse. The time- and temperature-dependent scattering patterns recorded with this setup can be reproduced with reasonably high fidelity with a model involving linear combinations of temperature-independent biomolecule scattering patterns and temperature-dependent scattering from the hydration shell and surrounding solvent. The biomolecule contribution to the scattering, which is acquired out to 5.6 $\AA$ - 1 , contains structural information down to chemical bond distances, and is sensitive to secondary structure. An additional benefit of acquiring scattering data over a broad range of temperatures is that it allows precise characterization of relative populations of species in equilibrium as well as thermodynamic properties of folding/unfolding, substrate binding, or oligomerization. 